\title{
Multivalued evolution equations with nonlocal initial conditions in Banach spaces
}

\author{
Sergiu AIZICOVICI \\ Department of Mathematics, Ohio University \\ Athens, OH 45701, USA \\ e-mail: aizicovi@math.ohiou.edu \\ Vasile STAICU \\ Department of Mathematics, Aveiro University \\ 3810-193 Aveiro, Portugal \\ e-mail: vasile@ua.pt
}

\begin{abstract}
We prove the existence of integral solutions to the nonlocal Cauchy problem

$$
u^{\prime}(t) \in-A u(t)+F(t, u(t)), 0 \leq t \leq T ; u(0)=g(u)
$$

in a Banach space $X$, where $A: D(A) \subset X \rightarrow X$ is m-accretive and such that $-A$ generates a compact semigroup, $F:[0, T] \times X \rightarrow 2^{X}$ has nonempty, closed and convex values, and is strongly-weakly upper semicontinuous with respect to its second variable, and $g: C([0, T] ; \overline{D(A)}) \rightarrow \overline{D(A)}$. The case when $A$ depends on time is also considered.

2000 Mathematics Subject Classification: 34G25, 35K90, 47J35.

Key words: Nonlocal Cauchy problem, evolution inclusion, m-accretive operator, compact semigroup, multifunction.
\end{abstract}

\section{Introduction}

This paper is concerned with the existence of solutions to the nonlocal Cauchy problem

$$
u^{\prime}(t) \in-A u(t)+F(t, u(t)), t \in I:=[0, T] ; u(0)=g(u),
$$

in a real reflexive, separable Banach space $X$. Here $A: D(A) \subset X \rightarrow X$ is a nonlinear (possibly multivalued) operator on $X$, such that $-A$ generates a compact 
semigroup of contractions on $\overline{D(A)}, F: I \times X \rightarrow 2^{X} \backslash\{\varnothing\}$ denotes a closed and convex valued multifunction, which is strongly-weakly upper semicontinuous with respect to its second variable, and $g: C(I ; \overline{D(A)}) \rightarrow \overline{D(A)}$. The time dependent counterpart of (1.1), namely

$$
u^{\prime}(t) \in-A(t) u(t)+F(t, u(t)), t \in I:=[0, T] ; u(0)=g(u),
$$

where $\{A(t), t \in I\}$ are m-accretive operators on $X$ and generate a compact evolution operator, is also discussed.

The study of nonlocal initial-value problems in Banach spaces was initiated by Byszewski [15], who considered an equation of the form (1.1) with $A$ linear, $F$ single valued, and $g$ of a special structure. Results on fully nonlinear abstract nonlocal Cauchy problem have been obtained in [2], [3], [4]. While we were concluding the writing up of this article, Xue's very recent work [28] has been brought to our attention. These papers are primarily concerned with equations governed by accretive operators and single-valued perturbations. To our knowledge, the only existing result for (1.1) with $A$ nonlinear and $F$ multivalued is Theorem 3.8 in [4], where $F$ is supposed to be closed-valued and lower semicontinuous in its second variable. On the other hand, finite dimensional versions of (1.1) (with $A=0$ ) appear in [13], [21], while abstract semilinear evolution inclusions with nonlocal initial conditions have been considered in [1], [9], [10], [11]. In particular, in [1], the problem (1.1) is analyzed under the assumption that $-A$ is the infinitesimal generator of a linear $C_{0}$-semigroup on $X, F$ is closed, convex valued and upper semicontinuous in its second argument, and $g$ is an integral operator.

The present work complements [4] by allowing $F(.,$.$) to be upper semi-$ continuous in its second variable (as opposed to lower semicontinuous) and also generalizes the theory of [1] to the case when $A$ is fully nonlinear.

The plan of the paper is as follows. Section 2 contains background material on multifunctions, m-accretive operators and evolution equations. The main results for the problems (1.1) and (1.2) are stated in Section 3, while the proofs are carried out in Section 4. Finally, Section 5 contains an example to which our abstract theory applies.

\section{Preliminaries}

For further background and details pertaining to this section, we refer the reader to [5], [6], [8], [14], [22], [24], [25], [26] and [27].

Let $X$ be a real Banach space with norm $\|\cdot\|$ and dual $\left(X^{*},\|\cdot\|_{*}\right)$. As usual, $2^{X}$ stands for the family of all nonempty subsets of $X$ and $\bar{\Omega}$ denotes the closure of a set $\Omega \in 2^{X}$. The collection of all nonempty closed (resp., nonempty, closed and convex) subsets of $X$ is denoted by $\mathcal{P}(X)$ (resp., $\mathcal{P}_{c}(X)$ ). Let $\sigma\left(X, X^{*}\right)$ be the weak topology on $X$, which is known to be a Hausdorff topology. The space $X$ endowed with the $\sigma\left(X, X^{*}\right)$ topology will be denoted by $X_{w}$. The duality mapping 
$J: X \rightarrow 2^{X^{*}}$ is defined by

$$
J(x)=\left\{x^{*} \in X^{*}: x^{*}(x)=\|x\|^{2}=\left\|x^{*}\right\|_{*}^{2}\right\}, \forall x \in X .
$$

If $X^{*}$ is uniformly convex, then $J$ is single-valued and uniformly continuous on bounded subsets of $X$. The so called upper semi-inner product on $X$, denoted by $\langle., .\rangle_{+}$is given by

$$
\langle y, x\rangle_{+}=\sup \left\{x^{*}(y): x^{*} \in J(x)\right\} .
$$

Let $I=[0, T]$, where $0<T<\infty$. We denote by $C(I, X)\left(\operatorname{resp} . L^{1}(I, X)\right)$ the Banach space of all continuous (resp. Bochner integrable) functions $u: I \rightarrow X$ with norm $\|u\|_{\infty}=\sup _{t \in I}\|u(t)\|$, (resp. $\left.\|u\|_{1}=\int_{0}^{T}\|u(t)\| d t\right)$.

Let $A$ be a (possibly multivalued) operator in $X$. We define the domain and respectively, the range of $A$, by

$$
D(A):=\{x \in X: A x \neq \varnothing\}, R(A):=\bigcup_{x \in D(A)} A x .
$$

The operator $A$ is called accretive if $\left\langle y^{\prime}-y, x^{\prime}-x\right\rangle_{+} \geq 0$ for all $x, x^{\prime} \in$ $D(A), y \in A x$ and $y^{\prime} \in A x^{\prime}$. If also $R(I d+\lambda A)=X$, for each $\lambda>0$, where $I d$ is the identity map on $X$, then $A$ is said to be $m$-accretive.

By a celebrated result of Crandall and Liggett [17], if $A$ is m-accretive, then $-A$ generates a semigroup of contractions $\{S(t): t \geq 0\}$ on $\overline{D(A)}$. The respective semigroup is said to be compact if $S(t)$ maps bounded subsets of $\overline{D(A)}$ into precompact subsets of $\overline{D(A)}$, for each $t>0$.

Let $A$ be m-accretive in $X$. For $u_{0} \in \overline{D(A)}$ and $f \in L^{1}(I, X)$ we consider the initial value problem:

$$
u^{\prime}(t) \in-A u(t)+f(t), t \in I ; u(0)=u_{0},
$$

whose solutions are meant in the sense of the following definition that is due to Bénilan [12]:

Definition 1 An integral solution to (2.1) is a continuous function $u: I \rightarrow \overline{D(A)}$ with $u(0)=u_{0}$, such that, for all $x \in D(A), y \in A x$ and all $0 \leq s \leq t \leq T$,

$$
\|u(t)-x\|^{2} \leq\|u(s)-x\|^{2}+2 \int_{s}^{t}\langle f(\tau)-y, u(\tau)-x\rangle_{+} d \tau .
$$

It is well known that equation (2.1) has a unique solution $u \in C(I, \overline{D(A)})$. The following proposition summarizes an important property of integral solutions:

Proposition 2 Let $u$ and $v$ be integral solutions of $(2.1)$ that correspond to $\left(u_{0}, f\right)$ and $\left(v_{0}, g\right)$ respectively (where $u_{0}, v_{0} \in \overline{D(A)}$ and $\left.f, g \in L^{1}(I, X)\right)$. Then

$$
\|u(t)-v(t)\| \leq\left\|u_{0}-v_{0}\right\|+\int_{0}^{t}\|f(s)-g(s)\| d s
$$

for all $t \in I$. 
Next, let $\{A(t), t \in I\}$ be a family of (possibly multivalued) operators on $X$, of domains $D(A(t))$, with $\overline{D(A(t))}=\bar{D}$ (independent of $t$ ) which satisfy the assumption:

$\left(H_{A(t)}\right) \quad(i) R(I d+\lambda A(t))=X$, for all $\lambda>0$ and $t \in I$,

(ii) there exists a continuous function $m_{1}: I \rightarrow X$ and a continuous nondecreasing function $m_{2}: \mathbb{R}^{+} \rightarrow \mathbb{R}^{+}\left(\mathbb{R}^{+}:=[0, \infty)\right)$ such that

$$
\begin{aligned}
& \left\langle y_{1}-y_{2}, x_{1}-x_{2}\right\rangle_{+} \geq \\
& -\left\|m_{1}(t)-m_{1}(s)\right\|\left\|x_{1}-x_{2}\right\| m_{2}\left(\max \left\{\left\|x_{1}\right\|,\|x\|_{2}\right\}\right),
\end{aligned}
$$

for all $x_{1} \in D(A(t)), y_{1} \in A(t) x_{1}, x_{2} \in D(A(s)), y_{2} \in A(s) x_{2}$ $0 \leq s \leq t \leq T$.

In particular, for each $t \in I$, the operator $A(t)$ is m-accretive. If $\left(H_{A(t)}\right)$ holds, then (see, e.g., [26]) the family $\{A(t), t \in I\}$ generates a so-called evolution operator $U(t, s)$ on $\bar{D}$ via the formula

$$
U(t, s) x=\lim _{n \rightarrow \infty} \prod_{i=1}^{n}\left(I d+\frac{t-s}{n} A\left(s+i \frac{t-s}{n}\right)\right)^{-1} x
$$

for all $x \in \bar{D}$ and all $0 \leq s \leq t \leq T$. The evolution operator $U$ is said to be compact if $U(t, s)$ maps bounded subsets of $\bar{D}$ into precompact subsets of $\bar{D}$, for all $0 \leq s<t \leq T$.

Consider the nonautonomous Cauchy problem

$$
u^{\prime}(t) \in-A(t) u(t)+f(t), t \in I ; u(0)=u_{0},
$$

where $A(t)$ satisfies $\left(H_{A(t)}\right), f \in L^{1}(I, X)$ and $u_{0} \in \bar{D}$.

Definition 3 An integral solution of (2.4) is a function $u \in C(I, \bar{D})$ satisfying $u(0)=u_{0}$ and the inequality

$$
\begin{aligned}
\|u(t)-x\|^{2}-\|u(s)-x\|^{2} \leq 2 & \int_{s}^{t}\left[\langle f(\tau)-y, u(\tau)-x\rangle_{+}\right. \\
& \left.+C\|u(\tau)-x\|\left\|m_{1}(\tau)-m_{1}(\theta)\right\|\right] d \tau
\end{aligned}
$$

for all $0 \leq s \leq t \leq T, \theta \in I, x \in D(A(\theta)), y \in A(\theta) x$, and $C=m_{2}(\max \{\|x\|$, $\left.\left.\|u\|_{\infty}\right\}\right)$, with $m_{1}$ and $m_{2}$ as in $\left(H_{A(t)}\right)(i i)$.

Recall that (2.4) has an unique integral solution for each $u_{0} \in \bar{D}$ and $f \in$ $L^{1}(I, X)$, provided that $\left(H_{A(t)}\right)$ is satisfied. Moreover, the following analog of Proposition 2 is true: 
Proposition 4 Let $\left(H_{A(t)}\right)$ be satisfied and let $u$ and $v$ be integral solutions of (2.4) corresponding to $\left(u_{0}, f\right)$ and $\left(v_{0}, g\right)$, respectively (with $u_{0}, v_{0} \in \bar{D}$ and $f$, $\left.g \in L^{1}(I, X)\right)$. Then the inequality (2.3) holds for all $t \in I$.

The remainder of this section is devoted to a brief review of multifunctions. In what follows, the Banach space $X$ will be assumed separable.

Let $(\Omega, \Sigma, \mu)$ be a measure space and $\Phi: \Omega \rightarrow 2^{X}$. We say that $\Phi$ is measurable if $\{\omega \in \Omega: \Phi(\omega) \cap C \neq \varnothing\} \in \Sigma$ for any closed subset $C$ of $X$. If $\Omega \subset \mathbb{R}^{n}$ $(n \geq 1)$, then $\Sigma$ is the $\sigma$-algebra of Lebesgue measurable subsets of $\Omega$.

A function $\varphi: \Omega \rightarrow X$ that satisfies $\varphi(\omega) \in \Phi(\omega), \mu-$ a.e. on $\Omega$, is called a selection of $\Phi$. We define $\mathcal{S}_{\Phi}^{1}$ as the set of all selections of $\Phi$ that belong to $L^{1}(\Omega, X)$.

Let $Y$ and $Z$ be Hausdorff topological spaces and let $\Psi: Y \rightarrow 2^{Z}$. For $A \in 2^{Z}$ we set

$$
\Psi^{-}(A):=\{y \in Y: \Psi(y) \cap A \neq \varnothing\}, \quad \Psi^{+}(A):=\{y \in Y: \Psi(y) \subset A\} .
$$

The multifunction $\Psi$ is said to be upper semi-continuous on $Y$ (u.s.c., for short) if the set $\Psi^{+}(A)$ is open in $Y$ for any open subset of $A$ of $Z$.(Equivalently, $\Psi$ is u.s.c. if $\Psi^{-}(C)$ is closed in $Y$ for each closed subset $C$ of $Z$ ).

If $Z=X_{w}$ and $\Psi: Y \rightarrow 2^{Z}$ is u.s.c. and closed valued, then, by ([22], Chapter 1, Proposition 2.17) $\Psi$ is closed, that is,

$$
G r(\Psi):=\{(y, z) \in Y \times Z: z \in \Psi(y)\}
$$

is closed in $Y \times Z$. Conversely, if $\Psi: Y \rightarrow \mathcal{P}(Z)$ is closed and locally compact (i.e., for each $y \in Y$, there exists a neighborhood $U$ of $y$ such that $\Psi(U)$ is precompact), then $\Psi$ is u.s.c. (see ([22], Chapter 1, Proposition 2.23).

We will be mainly concerned with multifunctions $F: I \times X \rightarrow 2^{X}$ satisfying

$\left(H_{F}^{1}\right)$ For each $x \in X, F(., x)$ is measurable,

$\left(H_{F}^{2}\right)$ For a.a. $t \in I, F(t,$.$) is upper semicontinuous from X$ into $X_{w}$.

The following special form of the Convergence Theorem in [5], p.60, as given in [27], p.120, will be used in the sequel:

Proposition 5 Let $F: I \times X \rightarrow \mathcal{P}_{c}(X)$ satisfy assumptions $\left(H_{F}^{1}\right)$ and $\left(H_{F}^{2}\right)$. Let $u_{n}, f_{n}: I \rightarrow X(n \in \mathbb{N})$ be measurable functions such that $u_{n}$ converges almost everywhere on $I$ to a function $u: I \rightarrow X$ and $f_{n}$ converges weakly in $L^{1}(I, X)$ to $f: I \rightarrow X$. If $f_{n}(t) \in F\left(t, u_{n}(t)\right)$, for all $n \in \mathbb{N}$ and almost all $t \in I$, then $f(t) \in F(t, u(t))$, a.e. on $I$. 
The final result of this section is the following:

Lemma 6 Let $F: I \times X \rightarrow \mathcal{P}(X)$ satisfy assumptions $\left(H_{F}^{1}\right)$ and $\left(H_{F}^{2}\right)$, and let $u: I \rightarrow X$ be a measurable function.

Then the multifunction $t \rightarrow F(t, u(t))$ is measurable. If also there exists $\varphi \in L^{1}\left(I, \mathbb{R}^{+}\right)$such that

$$
|F(t, u(t))|:=\sup \{\|w\|: w \in F(t, u(t))\} \leq \varphi(t) \text {, a.e. on } I,
$$

then $S_{F(., u(.))}^{1}$ is a nonempty and closed subset of $L^{1}(I, X)$. Moreover $S_{F(., u(.))}^{1}$ is convex, provided that $F$ is convex valued.

Proof. Since $u: I \rightarrow X$ is measurable, there exists a sequence $\left(u_{n}\right)_{n \in \mathbb{N}}$ of simple functions from $I$ to $X$, such that $u_{n}$ converges to $u$, a.e. on $I$, as $n \rightarrow \infty$. By $\left(H_{F}^{1}\right)$, the multifunction $t \rightarrow F\left(t, u_{n}(t)\right)$ is measurable for each $n \in \mathbb{N}$. Using $\left(H_{F}^{2}\right)$, Propositions 2.13 and 2.17 in [22], Chapter 1, and Theorem 8.2.4 in [6] (cf., also [6], p.41, 313) we conclude that

$$
F(t, u(t))=\bigcap_{n \geq 1} \overline{\bigcup_{k \geq n} F\left(t, u_{k}(t)\right)}
$$

and that $t \rightarrow F(t, u(t))$ is measurable, as a multifunction from $I$ to $X$. Then, by the Kuratowski-Ryll Nardzewski Theorem (see, e.g. [22], p.154), there exists a measurable function $v: I \rightarrow X$ such that $v(t) \in F(t, u(t)), t \in I$. If (2.5) holds, then $v \in L^{1}(I, X)$, hence $S_{F(., u(.))}^{1} \neq \varnothing$. Recalling that $F$ is closed valued, it is easily verified that $S_{F(., u(.))}^{1}$ is closed in $L^{1}(I, X)$. Finally, if $F$ also has convex values, then it is immediate that $S_{F(., u(.))}^{1}$ is convex, as well.

\section{Statement of results}

Throughout this section, $X$ is supposed to be a real separable Banach space with uniformly convex dual $X^{*}, I:=[0, T]$, with $0<T<\infty$, and $\mathbb{R}^{+}:=[0, \infty)$. We study the nonlocal initial value problem (1.1) under the following assumptions:

$\left(H_{A}\right) A$ is an m-accretive operator in $X$, such that $-A$ generates a compact semigroup $\{S(t): t \geq 0\}$ on $\overline{D(A)}$.

$\left(H_{g}\right) g: C(I, \overline{D(A)}) \rightarrow \overline{D(A)}$ is such that

$$
\|g(u)-g(v)\| \leq m\|u-v\|_{\infty}, \forall u, v \in C(I, \overline{D(A)})
$$

for some $m$ with $0<m<1$.

$\left(H_{F}\right) F: I \times X \rightarrow \mathcal{P}_{c}(X)$ satisfies: 
(i) $F(., x)$ is measurable for each $x \in X$,

(ii) $F(t,$.$) is upper semicontinuous from X$ to $X_{w}$ for a.a. $t \in I$,

(iii) there exists a function $\gamma: I \times \mathbb{R}^{+} \rightarrow \mathbb{R}^{+}$such that $\gamma(., r) \in L^{1}(I, \mathbb{R})$ for every $r \in \mathbb{R}^{+}, \gamma(t,$.$) is continuous and nondecreasing for a.a.$ $t \in I$ and

$$
\lim \sup _{r \rightarrow \infty} \frac{1}{r} \int_{0}^{T} \gamma(t, r) d t<1-m
$$

where $m$ is the same as in condition $\left(H_{g}\right)$, with the additional property that

$$
|F(t, x)|:=\sup \{\|w\|: w \in F(t, x)\} \leq \gamma(t,\|x\|)
$$

for a.a. $t \in I$, and all $x \in \overline{D(A)}$.

Definition 7 A function $u \in C(I, \overline{D(A)})$ is called an integral solution of the problem (1.1) if there exists $f \in L^{1}(I, X)$ with $f(t) \in F(t, u(t))$, a.e. on $I$, such that $u$ is an integral solution in the sense of of Definition 1, of (2.1) with $g(u)$ in place of $u_{0}$.

Our basic existence result is the following:

Theorem 8 Let assumptions $\left(H_{A}\right),\left(H_{g}\right)$ and $\left(H_{F}\right)$ be satisfied. Then the set of integral solutions of the problem (1.1) is a nonempty, compact subset of $C(I, X)$.

Next we are concerned with the existence of integral solutions to the problem (1.2), where the operators $A(t)$ satisfy $\left(H_{A(t)}\right)$, while $F$ and $g$ are subject to conditions $\left(H_{F}\right)$ and $\left(H_{g}\right)$, respectively, with the mention that in $\left(H_{g}\right), \overline{D(A)}$ is to be replaced by $\bar{D}$. The corresponding modified assumption $\left(H_{g}\right)$ will be denoted by $\left(H_{g}^{\prime}\right)$.

Definition 9 A function $u \in C(I, \bar{D})$ is said to be an integral solution of the problem (1.2) if there exists $f \in L^{1}(I, X)$ with $f(t) \in F(t, u(t))$, a.e. on $I$, such that $u$ is an integral solution, in the sense of of Definition 3, of (2.4), where $u_{0}$ is replaced by $g(u)$.

The following counterpart of Theorem 8 is valid:

Theorem 10 Let assumptions $\left(H_{A(t)}\right),\left(H_{g}^{\prime}\right)$ and $\left(H_{F}\right)$ be satisfied. Then the set of integral solutions of the problem (1.2) is a nonempty, compact subset of $C(I, X)$. 


\section{Proofs}

Proof of Theorem 8. Without loss of generality, we assume that $0 \in D(A)$. We start with the initial value problem

$$
u^{\prime}(t) \in-A u(t)+f(t), t \in I ; u(0)=g(u),
$$

where $f \in L^{1}(I, X), A$ is m-accretive in $X$, and $g$ satisfies $\left(H_{g}\right)$. By an integral solution to $(4.1)$, we mean a function $u \in C(I, \overline{D(A)})$ that satisfies the requirements of Definition 1 , with $g(u)$ in place of $u_{0}$.

We claim that the problem (4.1) has a unique integral solution that will be denoted by $u_{f}$. Indeed, for each $v \in C(I, \overline{D(A)})$, there exists a unique integral solution $u_{v}$ of the initial value problem

$$
u^{\prime}(t) \in-A u(t)+f(t), t \in I ; u(0)=g(v),
$$

By (2.3) and $\left(H_{g}\right)$, we have

$$
\left\|u_{v}(t)-u_{w}(t)\right\| \leq\|g(v)-g(w)\| \leq m\|v-w\|_{\infty}, \forall t \in I
$$

hence

$$
\left\|u_{v}-u_{w}\right\|_{\infty} \leq m\|v-w\|_{\infty}
$$

for all $v, w \in C(I, \overline{D(A)})$. Since $0<m<1$, (4.3) implies that $v \rightarrow u_{v}$ is a strict contraction in $C(I, \overline{D(A)})$. Therefore, by the Contraction Mapping Principle, the map $v \rightarrow u_{v}$ has a unique fixed point in $C(I, \overline{D(A)})$. Obviously, this is the unique integral solution of (4.1).

Next, we obtain an a-priori bound for all possible solutions of (1.1). Let $u$ be an integral solution of (1.1), in the sense of Definition 7. Accordingly, there exists $f \in L^{1}(I, X)$ with $f(t) \in F(t, u(t))$, a.e. on $I$, such that $u$ is an integral solution of the related problem (4.1).

Noting that each $x \in D(A)$ can be viewed as the integral solution of $(2.1)$ with $f(t)=y \in A x$ and $u_{0}=x$, we obtain with the help of Proposition 2 that

$$
\|u(t)-x\| \leq\|g(u)-x\|+\int_{0}^{t}\|f(s)-y\| d s, t \in I,
$$

for a fixed $x \in D(A)$ and $y \in A x$. From (4.4) it follows that

$$
\|u(t)\| \leq 2\|x\|+\|g(0)\|+T\|y\|+\|g(u)-g(0)\|+\int_{0}^{T}\|f(s)\| d s .
$$


Using $\left(H_{g}\right)$ and $\left(H_{F}\right)($ iii $)$ in $(4.5)$ we arrive at

$$
(1-m)\|u\|_{\infty} \leq C+\int_{0}^{T} \gamma\left(s,\|u\|_{\infty}\right) d s
$$

where $C=2\|x\|+\|g(0)\|+T\|y\|$. This in conjunction with (3.1) implies that there exists a finite positive constant $M$ (which is independent of $u$ ) such that

$$
\|u\|_{\infty} \leq M
$$

Indeed, if this is not the case, one can construct a sequence $\left(u_{n}\right)_{n \in N}$ of integral solutions of (1.1), such that $\left\|u_{n}\right\|_{\infty} \rightarrow \infty$, as $n \rightarrow \infty$. Then, (4.6) leads to

$$
(1-m) \leq \lim \sup _{n \rightarrow \infty} \frac{\int_{0}^{T} \gamma\left(s,\left\|u_{n}\right\|_{\infty}\right) d s}{\left\|u_{n}\right\|_{\infty}} \leq \lim \sup _{r \rightarrow \infty} \frac{1}{r} \int_{0}^{T} \gamma(t, r) d t,
$$

in contradiction with (3.1). Hence, a constant $M$ must exist such that (4.7) is satisfied by all integral solutions $u$ of (1.1). Let

$$
\varphi(t):=\gamma(t, M)
$$

and remark $\left(\right.$ cf. $\left(H_{F}\right)($ iii $\left.)\right)$ that $\varphi \in L^{1}(I, \mathbb{R})$. Combining $(3.2),(4.7)$ and (4.8) we get

$$
|F(t, u(t))| \leq \varphi(t) \text {, a.e. on } I,
$$

for each integral solution $u$ of (1.1). In view of (4.9), we may assume without lost of generality that

$$
|F(t, x)| \leq \varphi(t), \forall x \in X, \text { a.e. on } I \text {. }
$$

Otherwise, we replace $F(t, x)$ by $\widetilde{F}(t, x)=F\left(t, p_{M}(x)\right)$, where $p_{M}: X \rightarrow X$ is given by

$$
p_{M}(x)=\left\{\begin{array}{ccc}
x & \text { if } & \|x\| \leq M \\
M \frac{x}{\|x\|} & \text { if } & \|x\|>M
\end{array},\right.
$$

with $M$ as in (4.7). It is easily seen that all conditions in $\left(H_{F}\right)$ are satisfied with $\widetilde{F}$ in place of $F$. In particular, one uses the strong-weak upper semicontinuity of $F$ and the continuity of $p_{M}$ to verify $(i i)$ in $\left(H_{F}\right)$. As regards to $(i i i)$, simply remark that $\left\|p_{M}(x)\right\| \leq\|x\|, \forall x \in X$, so that

$$
|\widetilde{F}(t, x)| \leq \gamma\left(t,\left\|p_{M}(x)\right\|\right) \leq \gamma(t,\|x\|),
$$

since $\gamma$ is nondecreasing in its second variable. 
Assume that we have found an integral solution $\widetilde{u}$ of the problem (1.1) where $F$ has been replaced by $\widetilde{F}$. By $(4.7)$ it follows that

$$
\|\widetilde{u}(t)\| \leq M, \forall t \in I,
$$

and therefore $(\operatorname{cf} .(4.11)), p_{M}(\widetilde{u}(t))=\widetilde{u}(t)$. As a result, $\widetilde{F}(t, \widetilde{u}(t))=F(t, \widetilde{u}(t))$, so that $\widetilde{u}($.$) is an integral solution of our original problem (1.1). Summarizing,$ we will henceforth suppose that (4.10) holds.

We now introduce the set $K \subset L^{1}(I, X)$ by

$$
K=\left\{f \in L^{1}(I, X):\|f(t)\| \leq \varphi(t) \text {, a.e. on } I\right\} .
$$

Clearly, $K$ is nonempty, closed and convex. In addition, $K$ is uniformly integrable, hence, by [18], p.101, it is compact in $L_{w}^{1}(I, X)$, where $L_{w}^{1}(I, X)$ denotes the space $L^{1}(I, X)$ equipped with its weak topology. We also note (see Theorem V.6.3 in [19]) that $K$, endowed with the relative $L_{w}^{1}(I, X)$ topology is a metric space. Define the map $\mathcal{F}: K \rightarrow 2^{L^{1}(I, X)}$ by

$$
\mathcal{F}(f):=S_{F\left(., u_{f}(.)\right)}^{1}=\left\{v \in L^{1}(I, X): v(t) \in F\left(t, u_{f}(t)\right) \text { a.e. on } I\right\},
$$

where $u_{f}$ (.) denotes the integral solution of (4.1), for a given $f \in K$.

By $\left(H_{F}\right)$ and Lemma 6 , we conclude that $\mathcal{F}(f)$ has nonempty, closed and convex values. Moreover, in view of $(4.9), \mathcal{F}(K) \subset K$. We regard $K$ as a compact convex subset, denoted as $K_{w}$, of $L_{w}^{1}(I, X)$ and show that $\mathcal{F}$ is u.s.c. from $K_{w}$ into $2^{K_{w}}$. By Proposition 2.23 in [22], Chapter 1 (cf., also Theorem 2.5 in [25]) it is sufficient to prove that its graph $G r(\mathcal{F})$ is sequentially closed in $K_{w} \times K_{w}$.

Let $\left(f_{n}, v_{n}\right) \in \operatorname{Gr}(\mathcal{F})$ with $f_{n} \rightarrow f$ and $v_{n} \rightarrow v$ in $L_{w}^{1}(I, X)$, as $n \rightarrow \infty$. From $(4.12),(4.4),(4.5)$ and $\left(H_{g}\right)$, it follows that $\left\{u_{f_{n}}\right\}_{n \in \mathbb{N}}$ is bounded in $C(I, X)$, and consequently, $\left\{g\left(u_{f_{n}}\right)\right\}_{n \in \mathbb{N}}$ is bounded in $X$.

We now show that $\left\{u_{f_{n}}\right\}_{n \in \mathbb{N}}$ is precompact in $C(I, X)$. To accomplish this, we reason as follows. (A comparable argument was independently used by Xue [28], in a different context.)

Let $\mathcal{X}:=C(I, \overline{D(A)})$ and define the operator $L: \mathcal{X} \rightarrow \mathcal{X}_{0}$ by

$$
(L w)(t)=w(t)-S(t) g(w), \forall t \in I, w \in \mathcal{X},
$$

where $\mathcal{X}_{0}=\{u \in C(I, X): u(t)=w(t)-S(t) g(w), t \in I$, for some $w \in \mathcal{X}\}$ and $\{S(t): t \geq 0\}$ denotes the contraction semigroup generated by $-A$. $\operatorname{By}\left(H_{g}\right)$, it is easily verified that $L$ is one-to-one and onto, and that $L^{-1}$ is continuous on $\mathcal{X}_{0}$, see [28], Lemma 2.5. Accordingly, we write

$$
u_{f_{n}}(t)=L^{-1}\left(w_{f_{n}}\right)(t), t \in I, n \in \mathbb{N}
$$

where (cf. (4.14)),

$$
w_{f_{n}}(t)=u_{f_{n}}(t)-S(t) g\left(u_{f_{n}}\right) .
$$


On account of $\left(H_{A}\right)$, one can invoke [7] (see also [16], Theorem 2.1) to infer that $\left\{w_{f_{n}}(.)\right\}_{n \in \mathbb{N}}$ is precompact in $C([\delta, T], X)$ for any $0<\delta<T$. Next, since by (4.16),

$$
w_{f_{n}}(0)=g\left(u_{f_{n}}\right)-g\left(u_{f_{n}}\right)=0, \forall n \in \mathbb{N}
$$

the set $\left\{w_{f_{n}}(0)\right\}_{n \in \mathbb{N}}$ is trivially compact in $X$. To prove the equicontinuity of $\left\{w_{f_{n}}(.)\right\}_{n \in \mathbb{N}}$ at $t=0$, remark that $z_{n}()=.S() g.\left(u_{f_{n}}\right)$ can be viewed as an integral solution of

$$
z^{\prime}(t) \in-A z(t), t \in I ; z(0)=g\left(u_{f_{n}}\right) .
$$

Applying Proposition 2, we obtain

$$
\left\|w_{f_{n}}(t)-w_{f_{n}}(0)\right\|=\left\|u_{f_{n}}(t)-z_{n}(t)\right\| \leq \int_{0}^{t}\left\|f_{n}(s)\right\| d s, \forall t \in I .
$$

Recalling that $f_{n} \in K$ (see (4.12)), we conclude that

$$
\left\|w_{f_{n}}(t)-w_{f_{n}}(0)\right\| \leq \int_{0}^{t} \varphi(s) d s, \forall t \in I .
$$

This implies that $\left\{w_{f_{n}}(.)\right\}_{n \in \mathbb{N}}$ is equicontinuous at $t=0$, as desired. In summary, by the Ascoli-Arzelà Theorem (cf., e.g., [27], Theorem 1.3.1), it follows that $\left\{w_{f_{n}}(.)\right\}_{n \in \mathbb{N}}$ is precompact in $\mathcal{X}_{o}$. This, in conjunction with (4.15) and the continuity of $L^{-1}$ on $\mathcal{X}$, implies that $\left\{u_{f_{n}}(.)\right\}_{n \in \mathbb{N}}$ in precompact is $\mathcal{X}$ as claimed.

Without loss of generality, we can assume that

$$
u_{f_{n}} \rightarrow u \text { in } C(I, X) \text {, as } n \rightarrow \infty,
$$

for some $u \in C(I, X)$. In view of (4.17) and the fact that $v_{n} \rightarrow v$ weakly in $L^{1}(I, X)$ with $v_{n} \in \mathcal{F}\left(f_{n}\right)$ (that is (cf. (4.13)), $v_{n}(t) \in F\left(t, u_{f_{n}}(t)\right)$, a.e. on $I$ ), we can apply Proposition 5 to conclude that

$$
v(t) \in F(t, u(t)) \text {, a.e. on } I \text {. }
$$

Finally, since $X^{*}$ is uniformly convex, the duality map $J: X \rightarrow X^{*}$ is single valued and continuous. By (2.2) and the definition of $\langle., .\rangle_{+}$(see Section 2), we have

$$
\left\|u_{f_{n}}(t)-x\right\|^{2} \leq\left\|u_{f_{n}}(s)-x\right\|^{2}+2 \int_{s}^{t} J\left(u_{f_{n}}(t)-x\right)\left(f_{n}(\tau)-y\right) d \tau,
$$

for all $x \in D(A), y \in A x$ and all $0 \leq s \leq t \leq T$. Using (4.17), the fact that $f_{n} \rightarrow f$ weakly in $L^{1}(I, X)$, and the uniform continuity of $J$, we can pass to the limit as $n \rightarrow \infty$ in (4.19) to obtain

$$
\|u(t)-x\|^{2} \leq\|u(s)-x\|^{2}+2 \int_{s}^{t}\langle f(\tau)-y, u(\tau)-x\rangle_{+} d \tau,
$$


for all $x \in D(A), y \in A x$ and all $0 \leq s \leq t \leq T$. Recalling that $u_{f_{n}}(0)=g\left(u_{f_{n}}\right)$, and employing $\left(H_{g}\right)$ and $(4.17)$, we infer that

$$
u(0)=g(u) .
$$

From (4.20) and (4.21), it follows that $u=u_{f}$. This, together with (4.18), enables us to conclude that $v \in \mathcal{F}(f)$, which completes the proof of the the sequential closedness of $\operatorname{Gr}(\mathcal{F})$.

We can now invoke the Kakutani-Ky Fan Fixed Point Theorem [20] to deduce that there exists $\widehat{f} \in K$ such that $\widehat{f} \in \mathcal{F}(\widehat{f})$. By (4.13), it is obvious that the corresponding integral solution of the problem (4.1), denoted by $u_{\hat{f}}$, is an integral solution of the problem (1.1), in the sense of Definition 7. This shows that the set of integral solutions of the problem (1.1) is a nonempty subset of $C(I, X)$, which will be denoted by $\mathcal{S}$.

It remains to show that $\mathcal{S}$ is compact in $C(I, X)$. Let $\left(u_{n}\right)_{n \in \mathbb{N}}$ be a sequence in $\mathcal{S}$, hence $u_{n}=u_{f_{n}}$ for some $f_{n} \in K$, with $f_{n}(t) \in F\left(t, u_{n}(t)\right)$, a.e. on $I$. Recalling that $K$ is compact in $L_{w}^{1}(I, X)$ and arguing as before, we may assume (without changing the notation for subsequences) that $u_{n} \rightarrow u$ in $C(I, X), f_{n} \rightarrow f$ weakly in $L^{1}(I, X)$, as $n \rightarrow \infty$. We then conclude (compare to (4.17)-(4.20)) that $u=u_{f}$, with $f(t) \in F(t, u(t))$, a.e. on $I$. In other words, $u \in \mathcal{S}$ and the proof of Theorem 8 is complete.

Proof of Theorem 10. One follows, step by step, the proof of Theorem 8, with the mention that $A(t), \bar{D},\left(H_{A(t)}\right)$, Definition 3 and Proposition 4 are now used in place of $A, \overline{D(A)},\left(H_{A}\right)$, Definition 1 and Proposition 2, respectively. In addition, in (4.14), (4.16) and the definition of $z_{n}(t), S(t)$ is to be replaced by $U(t, 0)$. One must also adapt the theory in [24] (see in particular Theorems 2, 3,4 and Lemma 4) to obtain the needed compactness property of the solution map $\left(u_{0}, f\right) \rightarrow u$, where $u_{0}$ varies in a bounded subset of $\bar{D}, f$ lies in a uniformly integrable subset of $L^{1}(I, X)$ and $u$ denotes the corresponding integral solution of (2.4), and justify the passage to the limit in the time-dependent counterpart of (4.19) (cf. Definition 3). The details are left to the reader.

\section{An example}

In this section we illustrate the applicability of our abstract theory, by discussing the obstacle type problem

$$
\left\{\begin{array}{l}
\frac{\partial u}{\partial t}-\Delta u=v \text { on } I \times \Omega, \\
-\frac{\partial u}{\partial \nu} \in \beta(u) \text { on } I \times \Gamma, \\
u(0, x)=\int_{0}^{T} h(s, u(s, x)) d s \text { on } \Omega, \\
v \in L^{1}\left(I, L^{2}(\Omega)\right), 0 \leq v(t, x) \leq q(t, x, u(t, x)) \text { on } I \times \Omega .
\end{array}\right.
$$

Here $I=[0, T], \Omega$ is a bounded domain in $\mathbb{R}^{n}(n \geq 1)$ with a smooth 
boundary $\Gamma, x=\left(x_{1}, \ldots, x_{n}\right) \in \Omega, \beta$ is a multivalued operator in $\mathbb{R}, h: I \times \mathbb{R} \rightarrow \mathbb{R}$, $q: I \times \Omega \times \mathbb{R} \rightarrow \mathbb{R}^{+}$and $\partial / \partial \nu$ denotes the outward normal derivative to $\Gamma$.

We impose the following restrictions on the data of the problem (5.1) :

$\left(H_{\beta}\right) \beta$ is m-accretive in $\mathbb{R}$ with $0 \in \beta(0)$;

$\left(H_{h}\right) h: I \times \mathbb{R} \rightarrow \mathbb{R}$ satisfies:

(i) $h(., r)$ is measurable for each $r \in \mathbb{R}$ and $h(., 0) \in L^{1}(I, \mathbb{R})$,

(ii) $h(t,$.$) is continuous for a.a. t \in I$,

(iii) there exists $k \in L^{1}\left(I, \mathbb{R}^{+}\right)$such that

$$
|h(t, r)-h(t, \bar{r})| \leq k(t)|r-\bar{r}|
$$

for almost all $t \in I$ and all $r, \bar{r} \in \mathbb{R}$;

$\left(H_{q}\right) q: I \times \Omega \times \mathbb{R} \rightarrow \mathbb{R}^{+}$satisfies

(i) $q(., ., r)$ is measurable for each $r \in \mathbb{R}$,

(ii) $q(t, x,$.$) is continuous for a.a. (t, x) \in I \times \Omega$,

(iii) there exist two functions $a \in L^{1}\left(I, L^{2}(\Omega)\right)$ and $b \in L^{1}(I, \mathbb{R})$, such that, for almost all $(t, x) \in I \times \Omega$ and all $r \in \mathbb{R}$, one has $a(t, x)$, $b(t) \geq 0$ and

$$
q(t, x, r) \leq a(t, x)+b(t)|r|
$$

Let $X=L^{2}(\Omega, \mathbb{R})$, equipped with the norm $\|\cdot\|_{2}$ (defined by $\|u\|_{2}=$ $\left.\left(\int_{\Omega} u^{2}(x) d x\right)^{\frac{1}{2}}, \forall u \in L^{2}(\Omega, \mathbb{R})\right)$. This is a separable Hilbert space. Define the operator $A$ in $X$ by

$$
A u=-\triangle u, D(A)=\left\{u \in H^{2}(\Omega):-\frac{\partial u}{\partial \nu} \in \beta(u), \text { a.e. on } \Gamma\right\} .
$$

By $\left(H_{\beta}\right)$ and [27], Remark 2.6, it follows that $A$ is m-accretive in $X$, with $\overline{D(A)}=X$, and $-A$ generates a compact semigroup on $X$. Next we introduce the functional $g: C(I, X) \rightarrow X$ by

$$
g(u)(x)=\int_{0}^{T} h(s, u(s, x)) d s, \forall u \in C(I, X), \text { a.e. on } \Omega .
$$

From $\left(H_{h}\right)$ it follows that $g$ is well defined and

$$
\|g(u)-g(\bar{u})\|_{2} \leq\|k\|_{1}\|u-\bar{u}\|_{\infty}, \forall u, \bar{u} \in C(I, X) .
$$

Finally, let $F: I \times X \rightarrow 2^{X}$ be given by

$$
F(t, u)=\{v \in X: 0 \leq v(x) \leq q(t, x, u(x)) \text {, a.e. on } \Omega\} .
$$


Using $\left(H_{q}\right)$, we conclude that $F$ has nonempty, closed and convex values and $F(., u)$ is measurable for all $u \in X$; see [23], p.191 and [6], Theorem 8.14. Also

$$
|F(t, u)|=\sup \left\{\|w\|_{2}: w \in F(t, u)\right\} \leq\|a(t, .)\|_{2}+b(t)\|u\|_{2} .
$$

Moreover, Proposition 2.2.3 in [22], Chapter 1, and the analysis in [23], p.191 imply that $F(t,$.$) is u.s.c. from X$ into $X_{w}$ for a.a. $t \in I$. See also [25], Theorem 2.5 .

It is now obvious that with the identifications (5.2), (5.3), and (5.5), we can rewrite $(5.1)$, in the form $(1.1)$, in the space $X=L^{2}(\Omega, \mathbb{R})$. On account of the above discussion (cf. in particular (5.4) and (5.6)), we conclude that assumptions $\left(H_{A}\right),\left(H_{F}\right)$ and $\left(H_{g}\right)$ are satisfied, provided that

$$
\|k\|_{1}+\|b\|_{1}<1
$$

(Note that, by (5.4), (5.7) one can take $m=\|k\|_{1}$ in $\left(H_{g}\right)$, and that (5.6) and (5.7) imply that (3.1) and (3.2) hold with $\gamma(t, r)=\|a(t, .)\|_{2}+b(t) r$.

By a generalized solution of (5.1), we mean an integral solution of its abstract equivalent (1.1) in the space $X=L^{2}(\Omega, \mathbb{R})$, with the identifications $(5.2),(5.3)$, and (5.5), in the sense of Definition 7. A direct application of Theorem 8 yields:

Theorem 11 Let conditions $\left(H_{\beta}\right),\left(H_{h}\right),\left(H_{q}\right)$ and $(5.7)$ be satisfied. Then the set of generalized solutions of the problem (5.1) is a nonempty, compact subset of $C\left(I, L^{2}(\Omega, \mathbb{R})\right)$.

\section{Acknowledgment}

This paper was completed while the first author was visiting the University of Aveiro as an Invited Scientist. The hospitality and financial support of the host institution are gratefully acknowledged. The second author acknowledges the financial support from Foundation of Science and Technology (FCT), Portugal, under the project FEDER POCI/MAT/55524/2004.

\section{References}

[1] N. U. AHMED, Differential inclusions on Banach spaces with nonlocal state constraints, Nonlinear Funct. Anal. Appl. 16 (2001), 359-409.

[2] S. AIZICOVICI and Y. GAO, Functional differential equations with nonlocal initial conditions, J. Appl. Math. Stochastic Anal. 10 (1997), 145-156.

[3] S. AIZICOVICI and H. LEE, Nonlinear nonlocal Cauchy problems in Banach spaces, Appl. Math. Letters 18 (2005), 401-407. 
[4] S. AIZICOVICI and M. MCKIBBEN, Existence results for a class of abstract nonlocal Cauchy problems, Nonlinear Anal. 39 (2000), 649-668.

[5] J. P. AUBIN and A. CELLINA, Differential Inclusions, Springer, Berlin, 1984.

[6] J. P. AUBIN and H. FRANKOWSKA, Set-Valued Analysis, Birkhauser, Boston, 1990.

[7] P. BARAS, Compacité de l'opérateur $f \rightarrow u$ solution d'une équation nonlinéaire $(d u / d t)+A u \ni f, C$. R. Acad. Sci. Paris 286 (1978), 1113-1116.

[8] V. BARBU, Nonlinear Semigroups and Differential Equations in Banach Spaces, Noordhoff International Publ., Leyden, 1976.

[9] M. BENCHOCHRA, E. GATSORI and S. K. NTOUYAS, Existence results for semi-linear integro-differential inclusions with nonlocal conditions, Rocky Mountain J. Math. 34 (2004), 833-848.

[10] M. BENCHOCHRA, E. GATSORI and S. K. NTOUYAS, Multivalued semilinear neutral functional differential equations with nonconvex-valued righthand side, Abstr. Appl. Anal. 6 (2004), 525-541.

[11] M. BENCHOHRA, Nonlocal Cauchy problems for neutral functional differential and integrodifferential inclusions in Banach spaces, J. Math. Anal. Appl. 258 (2001), 573-590.

[12] P. BÉNILAN, Solutions integrales d'équations d'évolution dans un espace de Banach, C. R. Acad. Sci. Paris 274 (1972), 47-50.

[13] A. BOUCHERIF, Nonlocal Cauchy problems for first order multivalued differential equations, Electron. J. Differential Equations 47 (2002), 1-9.

[14] H. BRÉZIS, Opérateurs Maximaux Monotones et Semigroupes de Contractions dans les Espaces de Hilbert, North-Holland, Amsterdam, 1973.

[15] L. BYSZEWSKI, Theorems about the existence and uniqueness of solutions of a semilinear evolution nonlocal Cauchy problem, J. Math. Anal. Appl. 162 (1991), 494-505.

[16] R. CASCAVAL and I. I. VRABIE, Existence of periodic solutions for a class of nonlinear evolution equations, Rev. Mat. Complut. 7 (1994), 325-338.

[17] M. CRANDALL and T. LIGGETT, Generation of semigroups of nonlinear transformations in Banach spaces, Amer. J. Math. 93 (1971), 265-298.

[18] J. DIESTEL and J. UHL, Vector Measures, Amer. Math. Soc., Providence, R.I., 1977. 
[19] N. DUNFORD and J. T. SCHWARTZ, Linear Operators, Part I, Interscience, New York, 1958.

[20] K. FAN, Fixed-point and minimax theorems in locally convex topological linear spaces, Proc. Nat. Acad. Sci. U. S. A. 38 (1952), 121-126.

[21] E. GATSORI, S. K. NTOUYAS and Y. G. SFICAS, On a nonlocal Cauchy problem for differential inclusions, Abstr. Appl. Anal. 5 (2004), 425-434.

[22] S. HU and N. S. PAPAGEORGIOU, Handbook of Multivalued Analysis, Vol. I: Theory, Kluwer, Dordrecht, 1997.

[23] S. HU and N. S. PAPAGEORGIOU, Handbook of Multivalued Analysis, Vol. II: Applications, Kluwer, Dordrecht, 2000.

[24] A. G. KARTSATOS and K. Y. SHIN, Solvability of functional evolutions via compactness methods in general Banach spaces, Nonlinear Anal. 21 (1993), $517-535$.

[25] M. KISIELEWICZ, Differential Inclusions and Optimal Control, PWNPolish Scientific Publishers and Kluwer Academic Publishers, Warszawa and Dordrecht, 1991.

[26] N. H. PAVEL, Nonlinear Evolution Operators and Semigroups, Lecture Notes in Math. 1260, Springer, Berlin, 1987.

[27] I. I. VRABIE, Compactness Methods for Nonlinear Evolutions, Longman, Harlow, 1987.

[28] X. XUE, Nonlinear differential equations with nonlocal conditions in Banach spaces, Nonlinear Anal., 63 (2005), 575-586.

Received 6 April 2005; accepted 31 May 2005

To access this journal online: http://www.birkhauser.ch 